\title{
Type I Diabetes Delays Perfusion and Engraftment of 3D Constructs by Impinging on Angiogenesis; Which can be Rescued by Hepatocyte Growth Factor Supplementation
}

\author{
Wafa Altalhi (iD, ${ }^{1,2,6}$ Rupal Hatkar, ${ }^{1}$ James B. Hoying, ${ }^{3}$ Yasaman Aghazadeh, ${ }^{1}$ \\ and SARA S. NunES $1,2,4,5$
}

${ }^{1}$ Toronto General Hospital Research Institute, University Health Network, 101 College St., MaRS, TMDT 3-904, Toronto, ON M5G 1L7, Canada; ${ }^{2}$ Laboratory of Medicine and Pathobiology, University of Toronto, Toronto, Canada; ${ }^{3}$ Advanced Solutions Life Sciences, Manchester, NH 03101, USA; ${ }^{4}$ Institute of Biomaterials and Biomedical Engineering, University of Toronto, Toronto, Canada; ${ }^{5}$ Heart \& Stroke/Richard Lewar Centre of Excellence, University of Toronto, Toronto, Canada; and

${ }^{6}$ Massachusetts General Hospital, 55 Fruit Street, Boston, USA

(Received 11 January 2019; accepted 9 May 2019; published online 21 May 2019)

Associate Editor Stephanie Michelle Willerth oversaw the review of this article.

\begin{abstract}
Introduction - The biggest bottleneck for cell-based regenerative therapy is the lack of a functional vasculature to support the grafts. This problem is exacerbated in diabetic patients, where vessel growth is inhibited. To address this issue, we aim to identify the causes of poor vascularization in 3D engineered tissues in diabetes and to reverse its negative effects.
\end{abstract}

Address correspondence to Sara S. Nunes, Toronto General Hospital Research Institute, University Health Network, 101 College St., MaRS, TMDT 3-904, Toronto, ON M5G 1L7, Canada. Electronic mail: sara.vasconcelos@utoronto.ca

Sara S. Nunes is a Scientist at the University Health Network in the Toronto General Hospital Research Institute. She holds an Assistant Professor appointment at the Institute of Biomaterials Biomedical Engineering and a cross-appointment at the Department of Laboratory Medicine \& Pathobiology at the University of Toronto. Her translational research program aims to develop regenerative medicine strategies, and use bioengineering approaches to study cardiovascular diseases and for drug testing. Nunes obtained her $\mathrm{Ph} . \mathrm{D}$. from the State University of Rio de Janeiro, Brazil, and completed postdoctoral training under Dr. James Hoying, Ph.D. at the University of Louisville and later with Prof. Milica Radisic, Ph.D. at the University of Toronto. Dr. Nunes received several awards and fellowships for her work, including the prestigious Early Researcher Award from the Ministry of Research Innovation and Science in Canada and the Scientist Development Grant from the American Heart Association, USA. She has developed new vascularization techniques to support functional tissues for organ regeneration and is pioneering the work to create mature vessels with specific arterio-venous identities in 3D engineered tissues. Her work on human cardiac tissues-on-a-dish, named biowires, has opened a new area of research in human pluripotent stem cell-derived cardiomyocyte maturation and drug testing which catalyzed further mechanistic and translational research in this area worldwide. She holds funding from CIHR, NSERC, CFREF and JDRF-USA. She serves as a committee review member for Canadian Institutes of Health Research, and as Ad Hoc reviewer for NIH study section. This article is part of the 2019 CMBE Young Innovators specia issue.
Methods - We used 3D vascularized constructs composed of microvessel fragments containing all cells present in the microcirculation, embedded in collagen type I hydrogels. Constructs were either cultured in vitro or implanted subcutaneously in non-diabetic or in a type I diabetic (streptozotocin-injected) mouse model. We used qPCR, ELISA, immunostaining, FACs and co-culture assays to characterize the effect of diabetes in engineered constructs.

Results - We demonstrated in $3 \mathrm{D}$ vascularized constructs that perivascular cells secrete hepatocyte growth factor (HGF), driving microvessel sprouting. Blockage of $\mathrm{HGF}$ or $\mathrm{HGF}$ receptor signaling in $3 \mathrm{D}$ constructs prevented vessel sprouting. Moreover, HGF expression in $3 \mathrm{D}$ constructs in vivo is downregulated in diabetes; while no differences were found in HGF receptor, VEGF or VEGF receptor expression. Low HGF expression in diabetes delayed the inosculation of graft and host vessels, decreasing blood perfusion and preventing tissue engraftment. Supplementation of HGF in 3D constructs, restored vessel sprouting in a diabetic milieu.

Conclusion-We show for the first time that diabetes affects HGF secretion in microvessels, which in turn prevents the engraftment of engineered tissues. Exogenous supplementation

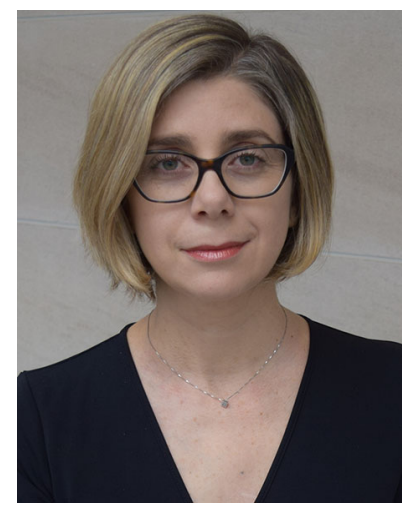


of $\mathrm{HGF}$, restores angiogenic growth in $3 \mathrm{D}$ constructs showing promise for application in cell-based regenerative therapies.

Keywords-Endothelial cell, Tissue engineering, Microvessel, Regenerative medicine, Revascularization, Angiogenesis, Hepatocyte growth factor, Blood perfusion, Diabetes, Anastomosis, Inosculation.

\section{INTRODUCTION}

Three-dimensional engineered tissues are a potential novel approach to replace damaged or dysfunctional tissues. ${ }^{29}$ However, a significant hindrance toward applying engineered tissues for regenerative medicine purposes is the lack of a functional vasculature ${ }^{15}$ to support the graft's metabolic needs, resulting in significant cell death and poor functional recovery. ${ }^{24}$ This issue is exacerbated in diseases that cause severe vascular damage and complications such as diabetes. ${ }^{1,21}$

Although diabetes has a 'pro-angiogenic' effect in the retina, ${ }^{20}$ it has been shown repeatedly to significantly decrease vessel sprouting in the peripheral circulation and to increase the chances of cardiovascular diseases such as myocardial infarction. ${ }^{21}$ People with diabetes also have decreased ability to recover from injuries and worse scarring. ${ }^{3}$ Therefore, for the development of efficient vascularization strategies that can produce mature, functional vessels in diabetes, it is important to study its effects in $3 \mathrm{D}$ engineered tissues.

Despite the significant advances made regarding our knowledge of how diabetes and the high glucose milieu affect vessel sprouting, ${ }^{8}$ we have yet to be able to generate an effective vascularization approach that can support large, 3D tissues in vivo. ${ }^{10,24,26}$ Our group has contributed to the growing vascular regeneration field by using microvessel fragments derived from adipose or brain tissues to generate $3 \mathrm{D}$ pre-vascularized tissues for in vivo applications. ${ }^{6}$ Our model includes the generation of $3 \mathrm{D}$ constructs consisting of microvessel fragments embedded in collagen type 1 gels. In this model, microvessel fragments grow, interconnect, and anastomose with the host circulation forming a functional, blood perfused vasculature with hierarchical organization and specific arteriovenous identities. ${ }^{18}$ This happens through a series of angiogenic adaptations occurring in a spatiotemporal fashion, and each step can be affected by diabetes. For instance, we were the first to show that diabetes affects vessel maturation and arterio-venous specification in $3 \mathrm{D}$ engineered constructs. ${ }^{1}$ Lack of vessel maturation was caused by the downregulation of the endothelialperivascular cell binding molecules Notch3 and Jagged 1 , and led to a decrease in perivascular cell coverage of the newly formed vascular bed.
To systematically assess the effects of diabetes and the potential for application of our 3D vascular regeneration model in regenerative medicine in the setting of diabetes, we interrogated here the effects of diabetes in vessel sprout formation in pre-vascularized constructs and whether diabetes' deleterious effects could be reversed. We demonstrate that hepatocyte growth factor (HGF) is downregulated in diabetes and by high glucose milieu decreasing vessel sprouting and delaying tissue engraftment and blood perfusion in vivo while exogenous HGF supplementation was found to restore vessel sprouting in engineered tissues in high glucose.

\section{MATERIALS AND METHODS}

\section{Animals}

All animal experiments were performed in compliance with institutional guidelines and were approved by the University Health Network Institutional Animal Care and Use Committee (protocols \#2420 and \#2427). Efnb2-reporter mice (arterial reporter): B6; 129S4-Efnb2 ${ }^{\text {tm2Sor }} / J$ mouse contain green fluorescent protein under control of the EphrinB2 promoter. $^{23}$ Heterozygous mice developed normally and were used as reporters for specific gene activity. Offspring were genotyped using standard PCR techniques according to Jackson Laboratories instructions. EphrinB2-reporter primers: $5^{\prime}-3^{\prime}$ AAG TTC ATC TGG ACC ACC $G$ and TCC TTG AAG AAG ATG GTG CG. Rag1 mice: B6.129S7-Rag $1^{\text {tm1Mom}} / J$-mouse lacking mature $\mathrm{T}$ and $\mathrm{B}$ cells, used as hosts for transplantation studies.

\section{Diabetic Mouse Model}

The model was generated according to the DiacComp protocol for high dose streptozotocin (STZ) diabetes induction. In short, 8- to 12-week-old male mice were injected once intraperitoneally with $150 \mathrm{mg} /$ $\mathrm{kg}$ STZ (Sigma Aldrich) in filter-sterilized $10 \mathrm{mM}$ sodium citrate buffer at $\mathrm{pH} 4.5$. Mice were fasted for $4 \mathrm{~h}$ before injections. Blood glucose measurements were performed 1 week after initial injection and every week thereafter for 5 weeks for donor mice, or every 2 weeks for Rag1 host mice. Mice were fasted for $4 \mathrm{~h}$ prior to tail blood collection. Mice that did not reach hyperglycemia (blood glucose $>15 \mathrm{mM}$ ) within 1 week of initial injection and remained non-diabetic, around $35 \%$ of total injected mice, were used as controls.

\section{Microvessel Isolation and 3D Construct Generation}

Mouse brain or rat epididymal fat pad microvessel fragments were isolated as previously described in Nunes et al. ${ }^{16}$ Briefly, tissue was minced and submitted 
to limited digestion with $2 \mathrm{mg} / \mathrm{ml}$ collagenase type I (4197) (Worthington) and $1 \mathrm{mg} / \mathrm{ml}$ DNAse-1 (DN25) (Sigma-Aldrich, St. Louis, MO, USA) in DPBS containing $0.1 \% \mathrm{BSA}$ at $37^{\circ} \mathrm{C}$ under agitation. Digested tissues underwent selective size filtration using 500 and $30 \mu \mathrm{m}$ filter screens. To generate $3 \mathrm{D}$ constructs, isolated microvessel fragments were suspended in type I collagen gels (3 mg/ml) (BD Biosciences) at 20,000 fragments $/ \mathrm{ml}$ of gel, cast into 48-well plates, and allowed to polymerize for $30 \mathrm{~min}$ at $37^{\circ} \mathrm{C}$. For in vivo neovascularization assays, constructs were implanted into subcutaneous skin pockets in Rag1 mice (one per flank) as previously described, ${ }^{5,17-19,24}$ and harvested at the indicated time points. Before harvesting constructs, mice were perfused intravenously with $100 \mu \mathrm{L}$ of $1 \mathrm{mg} / \mathrm{mL}$ of Rhodamine-Dextran (Sigma-Aldrich). Constructs were removed and imaged using an Olympus FluoView IX81 inverted confocal microscope (Olympus Life Science). For in vitro experiments, constructs were cultured with DMEM containing 10\% fetal bovine serum.

\section{Sprouting Blockage and Assessment}

Constructs grown in DMEM media with $10 \%$ FBS were supplemented with different concentrations of cMET inhibitor (SU11274) or $20 \mu \mathrm{g} / \mathrm{mL}$ of anti-HGF (SV114, R\&D). Control samples were treated with DMSO or IgG control antibodies. After 7 days in culture, sprouting was assessed by a blinded investigator as $0=$ no sprouting (not angiogenic) $; 1=$ low sprouting (1-2 sprouts/fragment; angiogenic); 2 = high sprouting (>3 sprouts/microvessel; highly angiogenic) as previously. ${ }^{5}$ For assessing sprouting index following HGF treatment at different concentrations, rat epididymal adipose tissue derived microvessel fragments were cultured in 3D constructs in either $5 \mathrm{mM}$ Glucose, $25 \mathrm{mM}$ D-glucose, or $25 \mathrm{mM}$ D-glucose supplemented with either $1.25,1.8,2.5$, or $3.1 \mathrm{nM}$ HGF. The media was changed after 4 and 6 days. Sprouting was assessed at days $7-9$ post-seeding.

\section{Cell Culture}

Human umbilical vein endothelial cells (HUVECs, ScienCell Research Laboratories), were seeded on $0.1 \%$ gelatin and grown in complete Endothelial Cell Media (ECM1001). Human smooth muscle cells (hSMCs) and human pericytes were grown in complete medium 231 (M231500, GIBCO) and pericyte media (PM1201, ScienCell Research Laboratories), respectively. All cells were maintained at $37{ }^{\circ} \mathrm{C}$ and $5 \% \mathrm{CO}_{2}$ and passaged when at $90 \%$ confluency using $0.25 \%$ trypsin-EDTA (GIBCO). The cells were used between passages 3-7.

\section{Growth Factor Treatment}

Fifty-thousand HUVECs $/ \mathrm{cm}^{2}$ were seeded into 6well plates in complete media and allowed to grow overnight when they were serum starved for $24 \mathrm{~h}$ with DMEM $+0.1 \%$ FBS. HUVECs were treated with $2.2 \mathrm{nM}$ of VEGF (293-VE, R\&D Systems) or $1.25 \mathrm{nM}$ HGF (294-HGN, R\&D Systems) for different time points. Then RNA isolation, cDNA synthesis, and qRT-PCR were performed as described below. HGF was supplemented in culture at the following concentrations: $1.25,1.8,2.5$, or $3.1 \mathrm{nM}$ in high glucose (25 mM D-glucose) media.

\section{Flow Cytometry}

HUVECs treated with $100 \mathrm{ng} / \mathrm{ml}$ of VEGF (293VE, R\&D Systems) or HGF (294-HGN, R\&D Systems) for $12 \mathrm{~h}$ were washed with PBS and detached with TrypLE (GIBCO, Thermo Fisher Scientific). Cells were then fixed with $1.5 \%$ PFA for $20 \mathrm{~min}$ at RT, blocked with $10 \%$ goat serum containing PBS, stained with anti-EphrinB2 (AF496-SP, R\&D Systems) and anti-EphB4 (sc-5536, Santa Cruz Biotechnology) followed by anti-goat-AF-647 and anti-rabbit-AF-488. Flow cytometry analysis was performed on a BD FACSCanto II. The data were analyzed with FlowJo.

\section{ELISA}

Media was collected at the indicated time points and the concentration of rodent HGF secreted from constructs was assessed using an HGF ELISA kit (Cat\# MHG00, R\&D Systems) according to manufacturer instructions. HGF secretion from human SMCs and pericytes was determined using the human-specific HGF ELISA kit Cat\# DHG00 (R\&D Systems).

\section{RNA Isolation and Quantitative RT-PCR ( $q R T-P C R$ )}

RNA was isolated using standard Trizol extraction. RNAbee (AMS Biotechnology, Abingdon, UK) was used for constructs and Ribozol (AMRESCO LLC, Solon, Ohio, USA) for cells. Chloroform was added at 1:5 of total sample volume. Samples were then centrifuged at $13,000 \mathrm{rpm}$ for $30 \mathrm{~min}$ to separate the aqueous phase. RNA was precipitated by adding an equal volume of isopropanol and centrifuging at $13,000 \mathrm{rpm}$ for $30 \mathrm{~min}$. Pellets were washed twice with $75 \%$ ethanol and then suspended in $35 \mu \mathrm{L}$ of water. DNAse treatment was done with DNAse I (New England Biolabs, Whitby, Canada) according to manufacturer instructions. A total of $1 \mu \mathrm{g}$ RNA was used for cDNA synthesis with the Qscript cDNA super mix reverse transcriptase kit (Quantabio, Beverly, MA, 
USA) according to manufacturer instructions. QRTPCR was set up in triplicate using the SYBR Green RT PCR master mix and a Roche 480 thermal cycler (Roche, Mississauga, Canada). Relative gene expression levels were calculated after normalization with a housekeeping gene (Tata binding protein, Tbp) and compared to control condition using the delta-deltaCT method. Details of the primer sequences used are provided in Supplementary Table 1.

\section{Statistical Analysis}

Data are expressed as mean \pm SEM. Statistical significance was calculated by one- or two-way ANOVA using Prism 6 software. A significance level of $p<0.05$ was considered statistically significant.

\section{RESULTS}

Intrinsically Secreted HGF is Required for Microvessel Sprouting in Engineered Tissues

In order to further characterize the angiogenic mechanisms taking place in the $3 \mathrm{D}$, pre-vascularized constructs, we tested whether HGF, a pro-angiogenic cytokine secreted from perivascular cells that binds to cMET on endothelial cells and induces their sprouting and proliferation, is secreted and if it has any influence on the sprouting angiogenesis of microvessel fragments. The data shown in (Fig. 1a) indicates that HGF is in fact released intrinsically from the constructs over time $(0 \pm 0, \quad 0.92 \pm 0.15, \quad 3.95 \pm 1.86, \quad 7.77 \pm 1.09 \mathrm{nM} ;$ mean \pm STE, $n=3$ ). Furthermore, blocking $\mathrm{HGF} / \mathrm{c}$ MET axis impairs sprouting angiogenesis (Fig. 1b), as adding an anti-HGF antibody or a c-MET chemical inhibitor (SU11274) significantly decreased microvessel fragment sprouting (Fig. 1b). These results indicate that HGF is driving the angiogenic sprouting in microvessel fragment-based $3 \mathrm{D}$ constructs.

\section{HGF is Secreted from Smooth Muscle Cells and Pericytes}

To determine which cell type in the $3 \mathrm{D}$ constructs is responsible for HGF secretion, we quantified HGF secretion from isolated perivascular cells in culture by ELISA. As shown in Fig. 2a, both smooth muscle cells (SMCs) and pericytes secreted HGF. Moreover, pericytes were shown to secrete $\sim 18 \times$ more HGF per cell than SMCs. Next, we stained vascularized constructs at day 5 post-isolation, a time point where vessel sprouting is abundant and HGF secretion is high. As shown in (Fig. 2b), HGF (red) co-localized with smooth muscle actin (green), but not endothelial cells (blue), thus confirming that HGF is secreted from perivascular cells.

\section{HGF Induces Ambiguous AV Marker Expression}

We have previously shown that the newly formed vessel sprouts in 3D constructs displayed an ambiguous arterio-venous (AV) identity-meaning that they co-expressed AV markers Ephrin-B2 and Eph-B4. ${ }^{18}$ Here, we tested whether treatment of endothelial cells with HGF, the angiogenic stimulus (a)

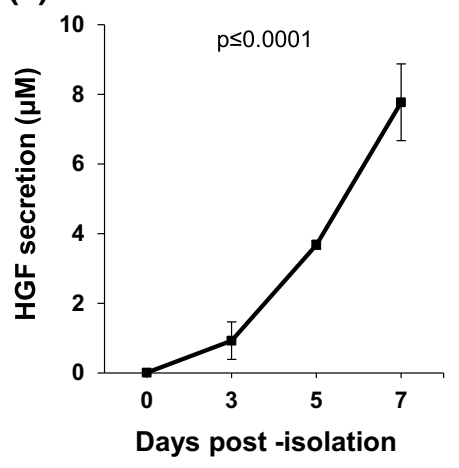

(b)

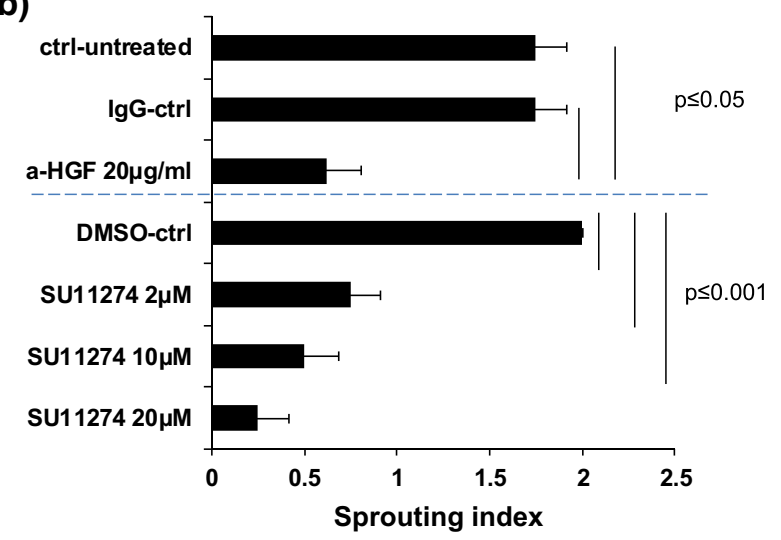

FIGURE 1. HGF is secreted by adipose-derived microvessel fragments in 3D constructs and mediates angiogenic sprouting. (a) In vitro analysis of HGF secretion from pre-vascularized engineered constructs. Pre-vascularized engineered constructs were maintained in $20 \%$ FBS-DMEM. Media was collected at the indicated time-points and HGF secretion was measured by ELISA (mean \pm SEM; one-way ANOVA, Bonferroni correction, $p \leq 0.0001$ vs. day 0 ). (b) Blockage of HGF/c-MET significantly reduces sprouting angiogenesis. Pre-vascularized constructs were maintained in $20 \%$ FBS-DMEM containing either anti-HGF antibody (aHGF), c-MET inhibitor (SU11274), IgG control, or DMSO (vehicle) for 7 days (mean \pm SEM; $n=3$ one-way ANOVA, Bonferroni correction, anti-HGF $p \leq 0.05$, SU11274, $p \leq 0.0001$ ). 
(a)

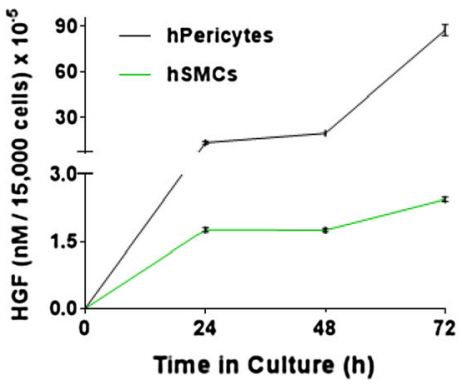

(b)

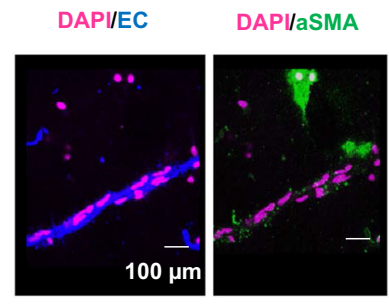

DAPI/HGF

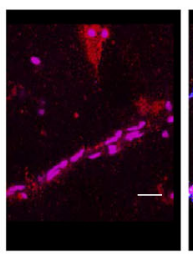

Overlay

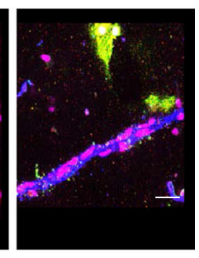

FIGURE 2. HGF is secreted from cultured perivascular cells. (a) Quantification of HGF secretion from smooth muscle cells or pericytes by ELISA (mean \pm SEM; two-way ANOVA, Bonferroni correction; $p \leq 0.0001$ ). (b) HGF expression by perivascular cells was confirmed in vitro in the pre-vascularized engineered constructs. Immunofluorescence staining of HGF at day 5 shows that HGF (red) co-localizes with alpha-smooth muscle cell actin (aSMA, perivascular cell marker, green) but not lectin (endothelial cell marker, blue).

(a)

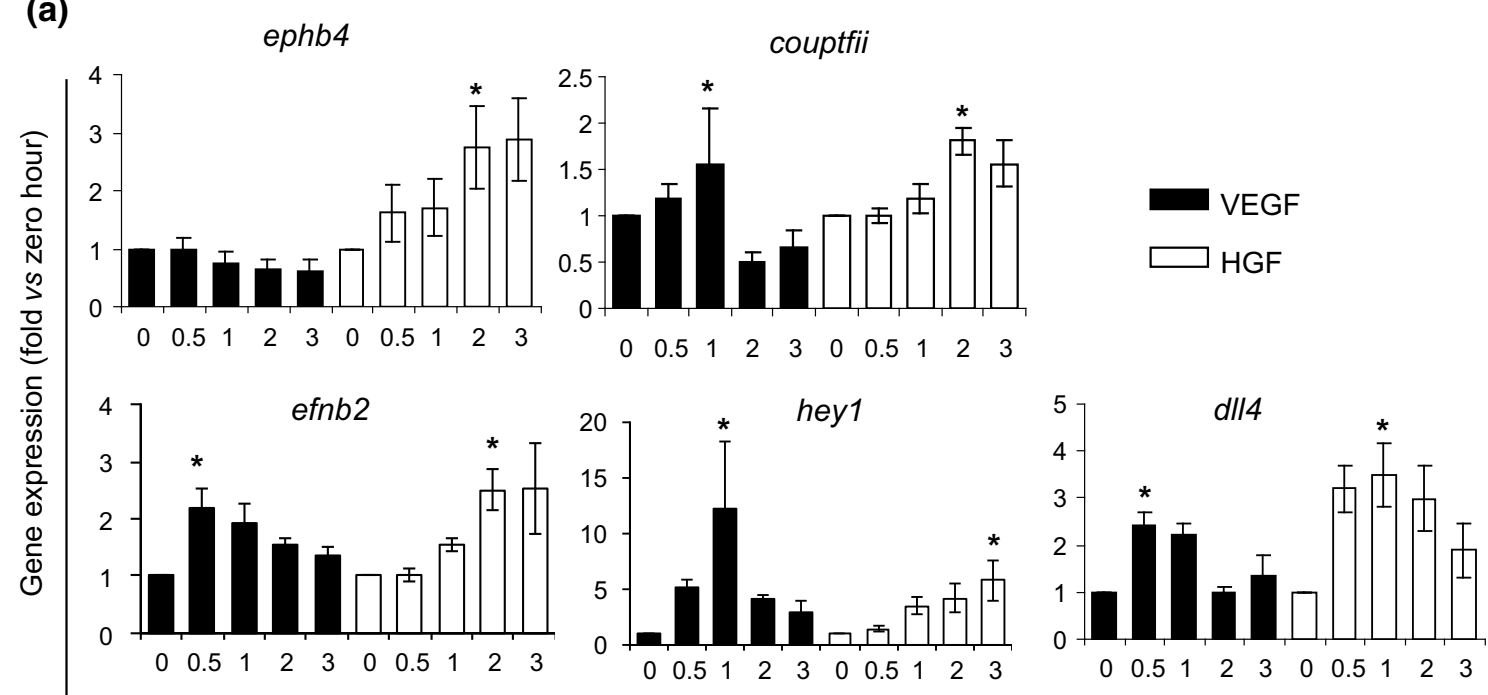

(b)

Time (hour)

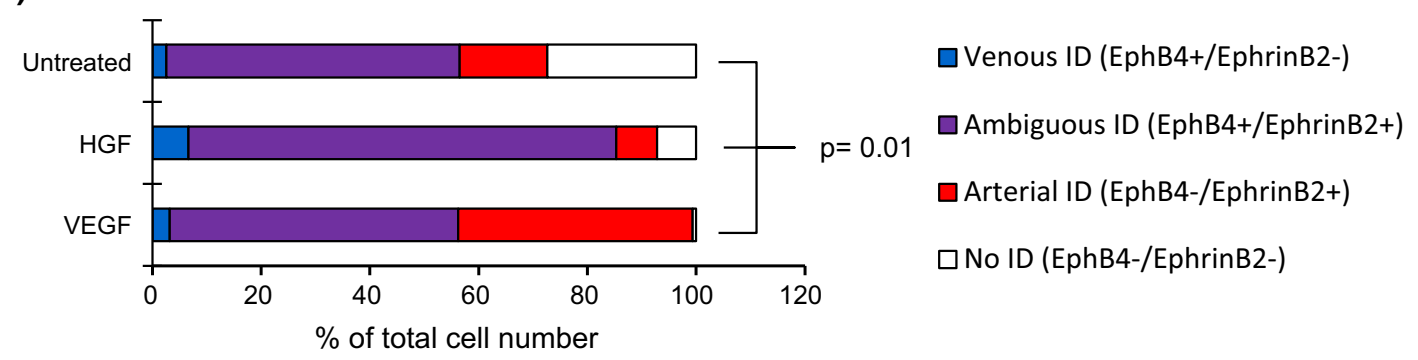

FIGURE 3. HGF induces ambiguous arterio-venous marker expression. (a) HUVECs were treated with either HGF or VEGF (arterial gene inducer, control). RNA was isolated at different time points post treatment. HGF induced expression of arterial and venous genes Hey1, Coup-tflI, EphrinB2, EphB4, and DII4 while VEGF induced expression of arterial genes Hey1, EphrinB2, and DII4 only (mean \pm SEM; $n=7$; one-way ANOVA, Dunnett's test, ${ }^{\star} p \leq 0.05$ vs. 0 h). (b) To confirm co-expression on the single-cell level, HUVECs were stimulated with HGF or VEGF for $12 \mathrm{~h}$, fixed, and stained with both anti-Ephrin-B2 and anti-Eph-B4 antibodies. Flow cytometry analysis showed that HGF increased the co-expression of Eph-B4 and Ephrin-B2 in the same endothelial cell compared to VEGF, and untreated conditions (mean \pm SEM; $n=3$; one-way ANOVA, Bonferroni correction, $p \leq 0.01$ of HGF vs. VEGF or untreated control).

intrinsically present in our pre-vascularized constructs, would lead to AV gene expression compatible with an ambiguous AV identity in vitro (AV identity loss). As opposed to VEGF, which induces the expression of arterial genes, HGF induces the upregulation of arterial markers Ephrin-B2 (efnb2), D114 
(dll4), and Heyl (dll4); and also venous markers EphB4 (ephb4) and Coup-TFII (couptfii) (Fig. 3a). To determine if the arterial and venous markers EphrinB2 and Eph-B4 were indeed co-expressed, as opposed to differentially induced in different cells resulting in the overall bulk increase in gene expression described in (Fig. 3a), we performed flow cytometry analysis of endothelial cells stimulated with either HGF or VEGF for $12 \mathrm{~h}$. As expected, treatment with VEGF led to a significant 2.7-fold increase in the percentage of cells expressing only the arterial marker EphrinB2 (Fig. 3b, red). Moreover, while there were no differences in the percentage of cells co-expressing the AV markers between the untreated controls $(53.9 \pm 8 \%)$ and VEGF-treated cells $(53 \pm 5.7 \%)$, treatment with HGF led to a $50 \%$ increase in the number of cells coexpressing Ephrin-B2 and Eph-B4 (Fig. 3b, purple, $78.6 \pm 5.4 \%$ ), representing an ambiguous AV identity. Treatment with HGF also led to a 2.5 -fold increase in the number of cells expressing only Eph-B4 (Fig. 3b, blue).
Diabetes Impairs HGF Secretion and Decreases Angiogenic Sprouting in Pre-Vascularized Engineered Tissues

To investigate the effects of diabetes in adipose-derived microvessels in pre-vascularized constructs, we performed in vivo implantation of $3 \mathrm{D}$ vascularized constructs into diabetic mice (STZ-induced, Fig. 4a, D/D) or non-diabetic (ND/ND, control) as previously. ${ }^{1}$ During days 6-9 postimplantation (Fig. 4b), we determined the expression of selected angiogenic growth factors and their respective receptors. $K l f 2$, a flow-responsive gene, was used as an indicator of blood perfusion and our control. Analysis of mRNA expression in pre-vascularized engineered tissues in both diabetic and non-diabetic mice revealed a significant decrease in $H g f$ but not $V e g f, c-M e t$, or Vegfr2 expression in 3D constructs in diabetes, as compared to constructs in non-diabetic controls (Fig. 4c). This suggests that diabetes might reduce angiogenic sprouting in pre-vascularized constructs by reducing HGF expression. Moreover, consistent with an increase in vessel perfusion described (a)

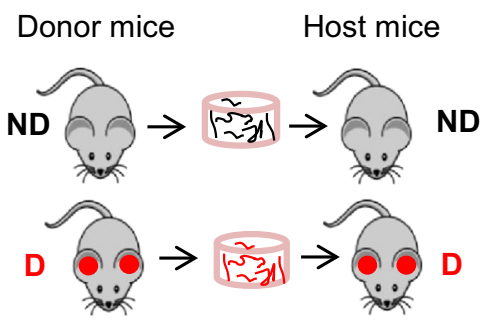

(b)

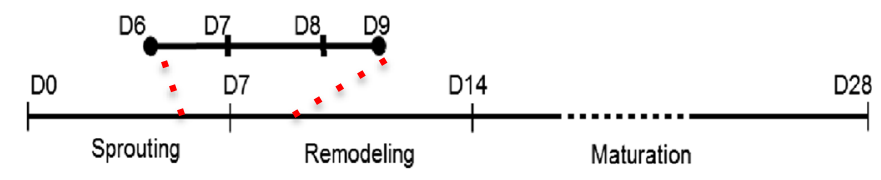

ND/ND D/D (c)

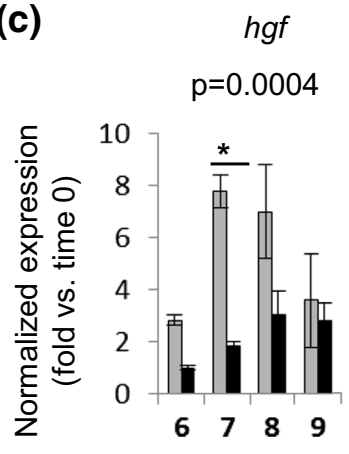

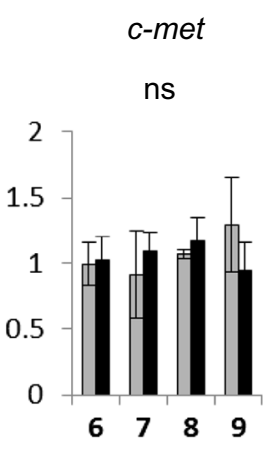

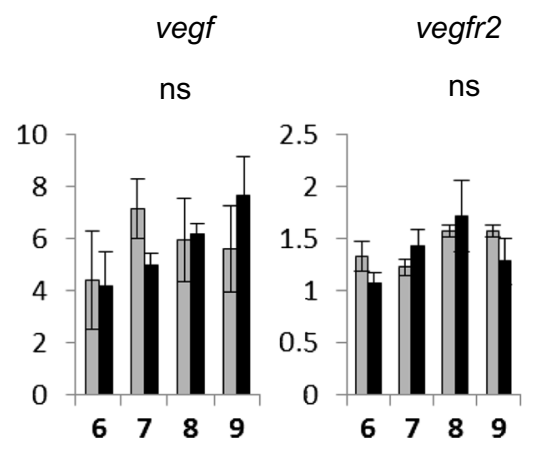

klf2

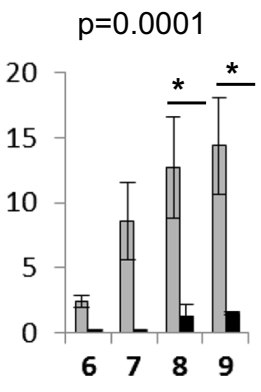

Time post implantation (Days)

FIGURE 4. Diabetes impairs HGF expression in pre-vascularized engineered constructs in vivo. (a) Schematic of vessel isolation: donor mice and implanted host mice. Microvessels from non-diabetic (ND) controls were implanted into ND hosts while vessels from STZ-induced diabetic mice with high glucose levels for a minimum of 5 weeks (diabetic; D) were implanted into $D$ hosts. (b) Experimental timeline. Explants were harvested at 6-9 days post-implantation for RNA isolation and analysis. (c) Analysis by qPCR gene expression during sprouting angiogenesis in both ND and D explants (gray and black, respectively). Data were normalized to housekeeping gene and to each time zero baseline (mean \pm SEM; $n=3-5$; one-way ANOVA, Bonferroni correction, ${ }^{*} p \leq 0.0001$; ns, non-significant). 
(a)

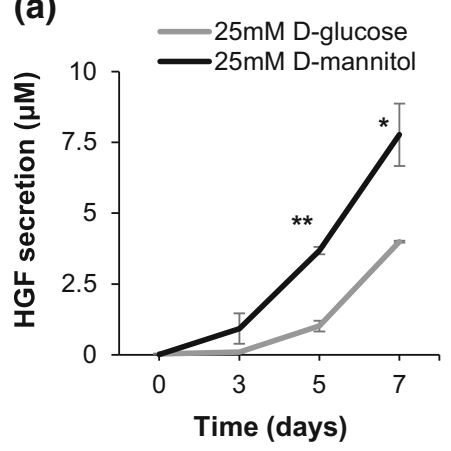

(b)

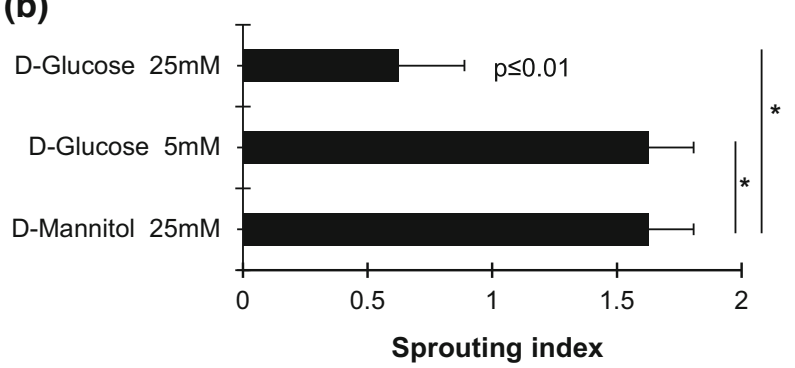

FIGURE 5. Diabetes impairs HGF secretion and decreases angiogenic sprouting in pre-vascularized engineered constructs. (a) In vitro analysis of HGF secretion from microvessel-based engineered constructs under high glucose conditions. Pre-vascularized tissues containing 20,000 microvessel fragments/ml were maintained in culture for 5 days in DMEM containing $10 \%$ FBS with either $25 \mathrm{mM}$ D-glucose or $25 \mathrm{mM}$ mannitol (control). HGF secretion was quantified by ELISA of culture supernatants (mean \pm SEM; $n=3$; two-way ANOVA, Bonferroni correction, $\left.{ }^{\star} p=0.3,{ }^{\star \star} p=0.0003\right)$. (b) Culture in high glucose (25 mM $\left.\mathrm{D}-\mathrm{glucose}\right) \mathrm{media}$ significantly inhibits sprouting as compared to control (25 mM mannitol) and low glucose (5 mM D-glucose). Data were coded to: $0=$ no sprouts; 1 = sprouting; $2=$ high sprouting (mean $\pm \mathrm{SEM} ; n=3$; one-way ANOVA, Bonferroni correction, ${ }^{*} p=0.01$ vs. $25 \mathrm{mM}$ D-mannitol or $5 \mathrm{mM}$ D-glucose).

previously ${ }^{7,12}$ Klf 2 expression increased in non-diabetic implants between days 6 and 9. However, Klf 2 induction was blunted in implants in diabetes (Fig. 4b), suggesting decreased blood perfusion.

To further characterize the role of diabetes in the 3D constructs, we tested the effects of high glucose in HGF secretion in vitro. HGF secretion was significantly reduced when constructs containing microvessel fragments from non-diabetic donors were grown in high glucose, compared to constructs grown in mannitol - which is devoid of metabolic activity and served as a hyperosmolar control (Fig. 5a). We then assessed the ability of microvessels in 3D constructs to sprout in high glucose conditions. We found that sprouting was significantly reduced in the presence of high glucose but not affected in mannitol controls (Fig. 5b).

\section{Diabetes Delays Engineered Tissue Engraftment and Vessel Perfusion}

We hypothesized that the reduction in HGF expression in early vasculatures in vivo (Fig. 4c) and in response to high glucose in vitro (Fig. 5a) would affect the integration of vascularized engineered tissue with the host. Therefore, we investigated vessel inosculation in pre-vascularized engineered tissues in the diabetic and control mice. We observed that implants from diabetic mice are pale compared to those from nondiabetic mice, which showed a general pink color indicative of blood perfusion (Fig. 6a). Confocal microscopy of the microvessels in pre-vascularized engineered constructs showed delayed connection to the recipient circulation in diabetes, with $0 \%$ of constructs explanted displaying any blood perfusion at day 7 and partial perfusion $(25 \%)$ at day 9 , compared to the ND/
ND condition where vessels showed $\sim 80 \%$ perfusion at day 7 and $100 \%$ perfusion at day 9 . Taken together with the HGF secretion data (Fig. 4), these findings reveal that reduced HGF caused delayed connection of microvessel fragments in pre-vascularized engineered implants to the recipient circulation.

\section{Exogenous HGF Restores Microvessel Sprouting in 3D Tissues in Diabetes in a Dose-Dependent Manner}

Given that soluble HGF is required for adipose-derived microvessel sprouting in $3 \mathrm{D}$ constructs and that HGF secretion is blunted by high glucose condition in diabetes, we proposed that addition of exogenous HGF would rescue microvessel sprouting in the engineered 3D constructs. To assess effect of exogenous HGF on microvessel sprouting, the 3D prevascularized constructs were cultured in $10 \%$ FBS in DMEM supplemented with either $5 \mathrm{mM}$ D-glucose (normoglycemia) or $25 \mathrm{mM}$ D-glucose (hyperglycemia) and increasing concentrations of HGF. Exogenous HGF supplementation restored microvessel sprouting in a dose-dependent manner (Fig. 7), with $3.1 \mathrm{nM} \mathrm{HGF}$ being similar to the normoglycemic, control condition.

\section{DISCUSSION}

The promise of $3 \mathrm{D}$ engineered tissues for regenerative medicine application hinges on the ability of vascularizing and perfusing large-scale tissues with blood upon in vivo implantation. ${ }^{24}$ There are a variety of factors that can play a role in tissue vascularization and ultimately affect the formation of functional vasculatures, including soluble growth factors, ${ }^{2}$ cell-cell ${ }^{9}$ 
(a)

$$
\text { Post-implantation }
$$

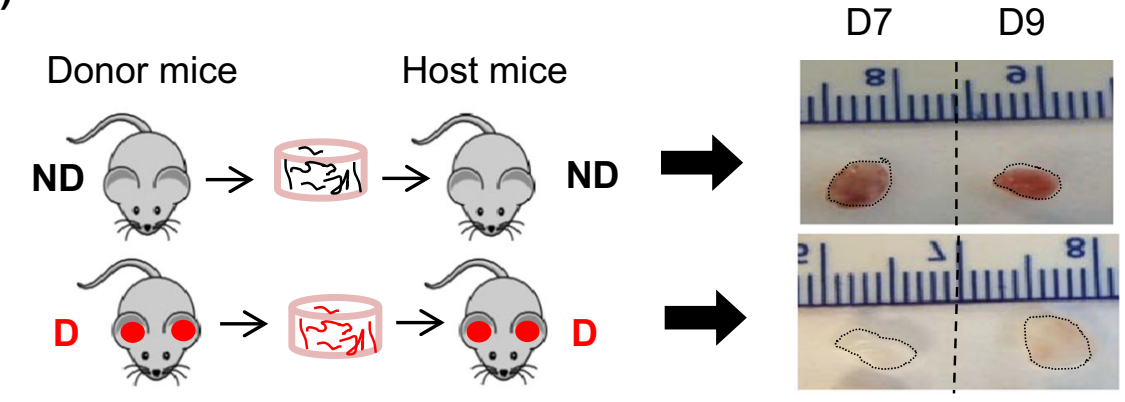

(b)

ND/ND

$\mathrm{D} / \mathrm{D}$

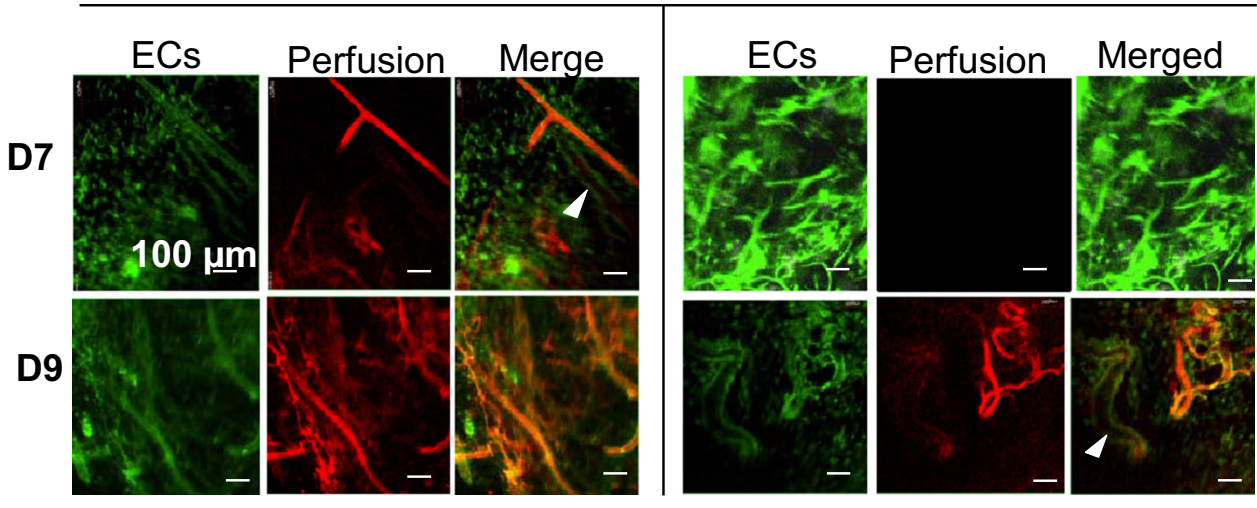

(c)

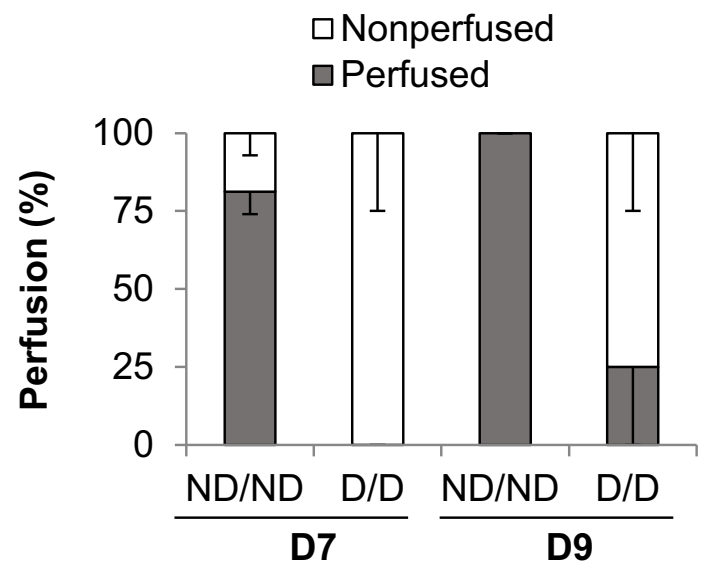

FIGURE 6. Diabetes delays the inosculation of graft and host vessels, decreasing blood perfusion and delaying tissue engraftment. (a) Microvessel fragments isolated from non-diabetic (ND) controls were implanted into ND hosts and microvessels isolated from STZ-induced diabetic mice with high glucose levels for a minimum of 5 weeks (diabetic; D) were implanted into D hosts. At day 7 or 9 post-implantation, mice were perfused intravenously with dextran-TRITC and the constructs were harvested. Representative gross appearance of harvested constructs. (b) Confocal microscopy images show delayed perfusion in D/D compared to ND/ND; $\boldsymbol{n}=3$. Arrowheads point to non-perfused vessels. (c) Quantification of the percentage of vessel perfusion on days 7 and 9 post-implantation (mean \pm SEM; $n=3$; one- way ANOVA, Bonferroni correction, $p=0.01$ for D/D vs. ND/ND at day 7 , $p=0.05$ for D/D vs. ND/ND at day 9 ).

and cell-matrix interactions, ${ }^{22}$ cell source, biophysical and biomechanical cues ${ }^{11}$ etc. We have previously shown that the VEGF-VEGF receptor signaling axis was not actively involved in the early vessel sprouting in the microvessel-based 3D, pre-vascularized constructs. ${ }^{5}$ Moreover, RNA analysis of explanted tissues showed steady levels of VEGF secretion over time ${ }^{18}$ without significant induction of VEGF expression, supporting a lack of VEGF-induced angiogenic sprouting in the microvessel-based 3D constructs. Here, intrinsically secreted HGF was found to promote the angiogenic sprouting of microvessel frag- 
(a)
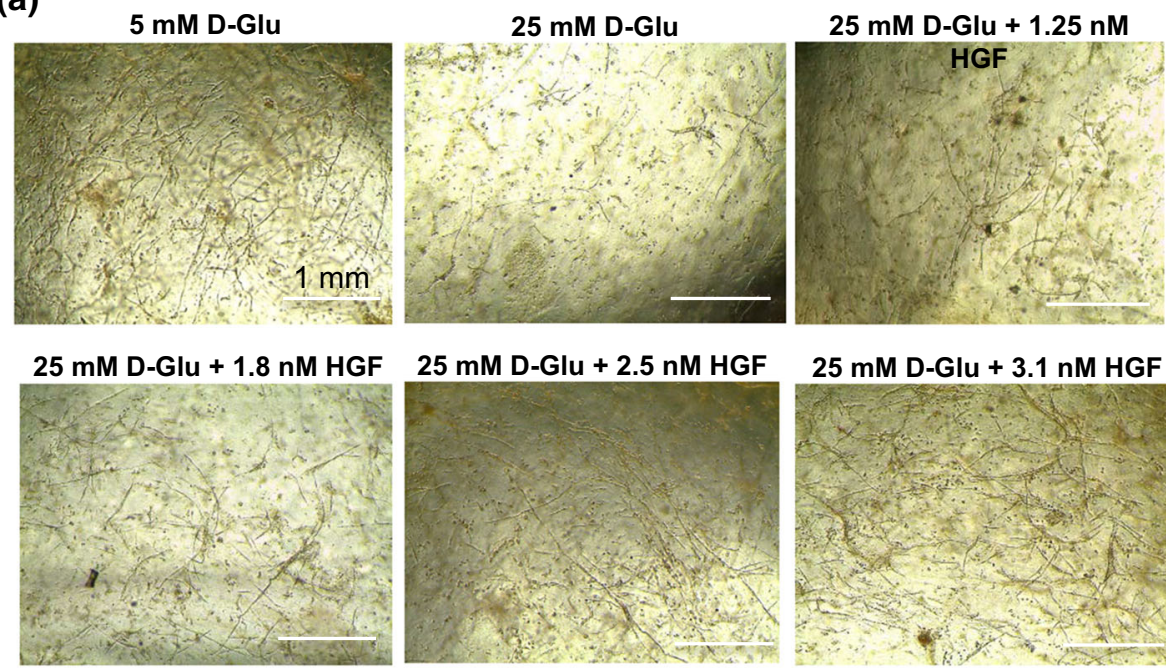

$25 \mathrm{mM}$ D-Glu + 3.1 nM HGF

(b)

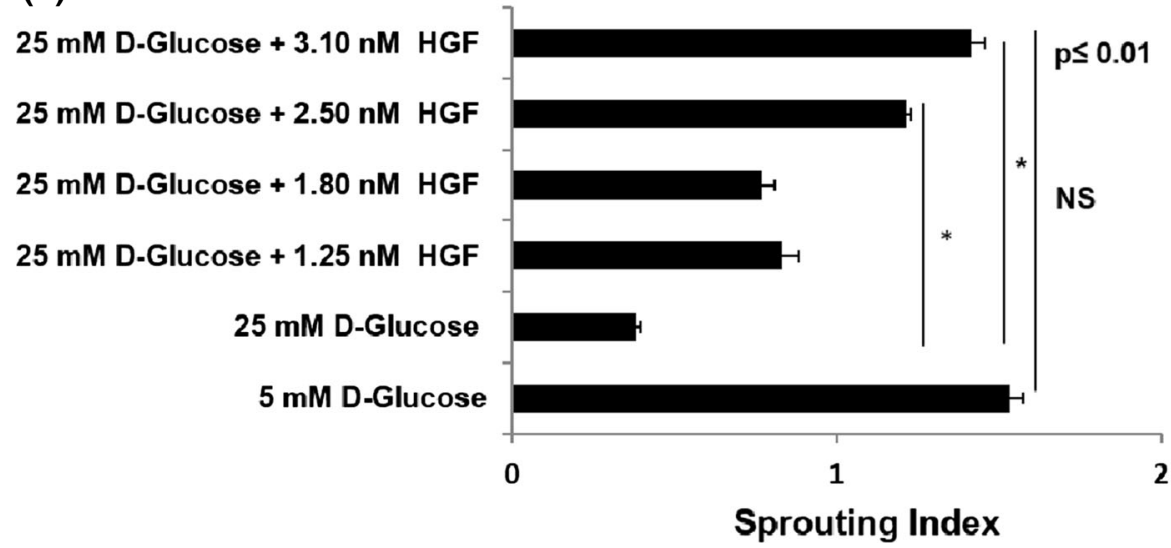

FIGURE 7. Exogenous HGF supplementation restores microvessel sprouting in 3D tissues in high glucose in a dose-dependent manner. (a) Representative bright field images of the 3D constructs taken 8 days post-seeding in $5 \mathrm{mM}$ or $25 \mathrm{mM}$ D-glucose (D-glu) with or without exogenous HGF supplementation. (b) Exogenous addition of HGF rescued the defective angiogenic sprouting caused by high glucose in a dose-dependent manner. 0 = low sprouting; 1 = medium sprouting; 2 = high sprouting (mean $\pm \mathrm{SEM}$; $n=3$; one-way ANOVA, Bonferroni correction, $p \leq 0.01$ ).

ments in 3D constructs, and that blocking the HGF-cMET axis prevented angiogenesis. HGF seems to be secreted by perivascular cells not closely associated with the vessel wall and that pericytes, present in abundance in the microvasculature, secrete 18-fold more HGF than smooth muscle cells in culture suggesting that pericytes drive the angiogenic response in the microvessel-based constructs. HGF is a wellknown angiogenic factor secreted by adipose-derived stromal vascular fraction cells and has been implicated in their role in tissue regeneration. ${ }^{13}$ Therefore, it is reasonable that intrinsically secreted $\mathrm{HGF}$ by the microvasculature has a significant role in the sprouting angiogenesis of these vessels. This is in contrast with predominant work in the literature where a role for VEGF is emphasized and suggests potential new avenues and application in engineered tissues for vascular regeneration. ${ }^{26}$
We have previously described that during normal angiogenic growth in 3D constructs, microvessel fragments that display specific arterial or venous identities underwent the loss of pre-existing arterio-venous identities in newly formed, immature sprouts. ${ }^{18}$ The key role of HGF in driving vessel sprouting in the 3D constructs suggested that HGF could be responsible for the loss of arterio-venous identity and the ambiguous identity observed. ${ }^{24}$ Indeed, we found that HGF induced the co-expression of both arterial and venous markers in human endothelial cells, resulting in an ambiguous arterio-venous identity. This is in contrast with VEGF that, as expected given the known role of VEGF in arteriogenesis, ${ }^{28}$ induced only the expression of genes and proteins of arterial identity only, and coincided with the growth of new sprouts in microvessels. 
Disease-specific factors such as high glucose, which is the hallmark of type I diabetes, can introduce several constrains when trying to create a functional vasculature for regenerative medicine applications. ${ }^{1,21,25}$ With this in mind we set out to investigate the effects of the diabetic milieu in the early sprouting and anastomosis of microvessels in $3 \mathrm{D}$ engineered constructs with the host vasculature. No significant changes in the expression of Vegf, c-Met, or Vegfr 2 was observed in pre-vascularized constructs in vivo in diabetic animals. Instead, we observed that HGF induction early postimplantation was blunted by diabetes and high glucose, which in turn decreased vessel sprouting. These findings are consistent with previous reports of reduced vessel sprouting as a complication observed in diabetes that results in decreased wound healing by rendering endothelial cells unresponsive to angiogenic stimuli by either reducing the expression of angiogenic growth factors or their receptors., ${ }^{3,4}$ Expression of Klf2 increased between days 6 and 9 in constructs implanted in healthy animals but not in ones implanted into diabetic mice. Klf2 is a flow-sensitive gene that functions to decrease vascular sprouting and promote vascular stability and maturation. ${ }^{7,14}$ Therefore, it is logical that $\mathrm{Kfl} 2$ is induced when the vasculature in the grafts inosculates with the host vessels and blood flow begins. ${ }^{12,16}$ The lack of upregulation of $K l f 2$ expression in diabetic conditions suggests the absence or delay of blood perfusion in the grafts. Indeed, we found that although vessels in the constructs in control conditions were $80 \%$ perfused by day 7 post-implantation, in diabetic conditions no perfusion was observed at this time point. By day 9, all vessels displayed blood perfusion in the grafts in control conditions but only $28 \%$ of perfusion was observed in diabetic conditions. The delay in blood perfusion observed was likely caused by the poor sprouting caused by low HGF levels which in turn delayed inosculation of host and construct vessels. This is likely to contribute to the defects in vessel maturation and arterio-venous specification we described previously since delayed blood perfusion will delay vessel remodeling, which starts after integration to the host vasculature, by promoting a decrease/delay perivascular cell recruitment.

Our group was the first to demonstrate that diabetes affects vessel maturation in a perivascular recruitmentdependent fashion in $3 \mathrm{D}$ engineered tissues and that controlling blood glucose levels led to normal vessel maturation and perivascular recruitment. This happens despite the chronical exposure of isolated microvessels to diabetes prior to isolation, suggesting that there were no long-term, memory effects. Here we have shown that exogenous supplementation of HGF in vitro can reverse vessel sprouting defects in diabetes, suggesting that this strategy could be used for pro- moting vessel growth in diabetes if glucose levels cannot be normalized. Indeed, others have shown that HGF is a potent angiogenic growth factor in mouse, rat, and rabbit ischemia models, including high-risk conditions such as diabetes ${ }^{27}$ and its application in humans has been investigated in clinical trials for ischemia.

Diabetes is known to affect vessel formation, hindering the utilization of vascularization strategies for tissue engineering in a large patient cohort that requires revascularization for regenerative medicine applications. ${ }^{15}$ Further studies are required to investigate if the delay in inosculation caused by diabetes observed here could be overcome by increasing vessel density in the constructs, which should increase HGF levels and the speed at which inosculation occurs (unpublished). Moreover, we do not know how delayed blood perfusion will affect different types of parenchymal cells that can be used in 3D tissue engineering. This will likely be dependent on the type of parenchymal cell used and their resistance to death in hypoxic environments -i.e., highly metabolic demanding cells such as cardiomyocytes will likely undergo apoptosis sooner than lower metabolic demanding cells. Regardless, our studies suggest that despite the deleterious effects of diabetes in vessel formation in microvessel-based $3 \mathrm{D}$ constructs, this approach could be applied in regenerative medicine if blood glucose levels can be controlled, which is standard practice with diabetes to prevent long-term cardiovascular damage, or by supplementing the constructs with exogenous HGF.

\section{ELECTRONIC SUPPLEMENTARY MATERIAL}

The online version of this article (https://doi.org/10. 1007/s12195-019-00574-3) contains supplementary material, which is available to authorized users.

\section{ACKNOWLEDGMENTS}

This work was supported by grants from the Canadian Institutes of Health Research (CIHR), Institute of Circulatory and Respiratory Health (137352 and PJT153160) and the Heart and Stroke Foundation of Canada (G-14-0006265) to S.S.N; NIH Grant (EB007556) to J.B.H. A Discovery grant from the Natural Sciences and Engineering Research Council (RGPIN 06621-2017) and an Early Researcher Award from the Ministry of Research, Innovation and Science (ER17-13-149) to S.S.N. supported R.H. 


\section{AUTHORS CONTRIBUTION}

SSN designed the experiments, coordinated the project and contributed to the writing of the manuscript. JBH supported HGF blockage assays. WA designed and performed experiment and contributed to manuscript writing. $\mathrm{RH}$ contributed to performing experiments and writing manuscript and YA contributed to performing experiments.

\section{CONFLICT OF INTEREST}

J.B.H. is an inventor on a patent regarding the use of adipose-derived microvessels and has equity interest with Advanced Solutions Life sciences, which is commercializing isolated microvessel technology. A version of this technology was used as an experimental model in this manuscript. This equity was obtained after the work for this manuscript was completed. W.A., R.H., Y.A. and S.S.N. declare no conflict of interest.

\section{ETHICAL STANDARDS}

All animal studies were carried out in accordance with Institutional guidelines and approved by the Animal Care Committee at the University Health Network (ID 2420 and 2427). No human subjects were used in this study.

\section{REFERENCES}

${ }^{1}$ Altalhi, W., X. Sun, J. M. Sivak, M. Husain, and S. S. Nunes. Diabetes impairs arterio-venous specification in engineered vascular tissues in a perivascular cell recruitment-dependent manner. Biomaterials 119:23-32, 2017.

${ }^{2}$ Blanco, R., and H. Gerhardt. VEGF and notch in tip and stalk cell selection. Cold Spring Harb. Perspect. Med. 3:a006569, 2013. https://doi.org/10.1101/cshperspect.a006 569

${ }^{3}$ Brem, H., and M. Tomic-Canic. Cellular and molecular basis of wound healing in diabetes. J. Clin. Invest. 117:1219-1222, 2007. https://doi.org/10.1172/JCI32169.

${ }^{4}$ Cao, L., et al. Modulating notch signaling to enhance neovascularization and reperfusion in diabetic mice. Biomaterials 31:9048-9056, 2010. https://doi.org/10.1016/j.bi omaterials.2010.08.002.

${ }^{5}$ Chang, C. C., et al. Angiogenesis in a microvascular construct for transplantation depends on the method of chamber circulation. Tissue Eng. Part A 16:795-805, 2010. https://doi.org/10.1089/ten.TEA.2009.0370.

${ }^{6}$ Chang, C. C., et al. Determinants of microvascular network topologies in implanted neovasculatures. Arterioscler. Thromb. Vasc. Biol. 32:5-14, 2012.

${ }^{7}$ Dekker, R. J., et al. Endothelial KLF2 links local arterial shear stress levels to the expression of vascular tone-regulating genes. Am. J. Pathol. 167:609-618, 2005. https://doi. org/10.1016/S0002-9440(10)63002-7.
${ }^{8}$ Eelen, G., P. de Zeeuw, M. Simons, and P. Carmeliet. Endothelial cell metabolism in normal and diseased vasculature. Circ. Res. 116:1231-1244, 2015. https://doi.org/ 10.1161/CIRCRESAHA.116.302855.

${ }^{9}$ Gaengel, K., G. Genove, A. Armulik, and C. Betsholtz. Endothelial-mural cell signaling in vascular development and angiogenesis. Arterioscler. Thromb. Vasc. Biol. 29:630 638, 2009. https://doi.org/10.1161/ATVBAHA.107.161521.

${ }^{10}$ Krishnan, L., et al. Manipulating the microvasculature and its microenvironment. Crit. Rev. Biomed. Eng. 41:91-123, 2013.

${ }^{11}$ le Noble, F., et al. Flow regulates arterial-venous differentiation in the chick embryo yolk sac. Development 131:361-375, 2004. https://doi.org/10.1242/dev.00929.

${ }^{12}$ Lee, J. S., et al. Klf2 is an essential regulator of vascular hemodynamic forces in vivo. Dev. Cell 11:845-857, 2006. h ttps://doi.org/10.1016/j.devcel.2006.09.006.

${ }^{13}$ Nakamura, T., and S. Mizuno. The discovery of hepatocyte growth factor (HGF) and its significance for cell biology, life sciences and clinical medicine. Proc. Jpn. Acad. Ser. B Phys. Biol. Sci. 86:588-610, 2010.

${ }^{14}$ Nicoli, S., et al. MicroRNA-mediated integration of haemodynamics and Vegf signalling during angiogenesis. Nature 464:1196-1200, 2010. https://doi.org/10.1038/nature08889.

${ }^{15}$ Novosel, E. C., C. Kleinhans, and P. J. Kluger. Vascularization is the key challenge in tissue engineering. Adv. Drug Deliv. Rev. 63:300-311, 2011. https://doi.org/10.1016/j.add r.2011.03.004.

${ }^{16}$ Nunes, S. S., et al. Implanted microvessels progress through distinct neovascularization phenotypes. Microvasc. Res. 79:10-20, 2010.

${ }^{17}$ Nunes, S. S., et al. Angiogenic potential of microvessel fragments is independent of the tissue of origin and can be influenced by the cellular composition of the implants. Microcirculation 17:557-567, 2010.

${ }^{18}$ Nunes, S. S., et al. Generation of a functional liver tissue mimic using adipose stromal vascular fraction cell-derived vasculatures. Sci. Rep. 3:2141, 2013.

${ }^{19}$ Nunes, S. S., et al. Generation of a functional liver tissue mimic using adipose stromal vascular fraction cell-derived vasculatures. Sci. Rep. 3:2141, 2013.

${ }^{20}$ Rangasamy, S., R. Srinivasan, J. Maestas, P. G. McGuire, and A. Das. A potential role for angiopoietin 2 in the regulation of the blood-retinal barrier in diabetic retinopathy. Invest. Ophthalmol. Vis. Sci. 52:3784-3791, 2011. https://doi.org/10.1167/iovs.10-6386.

${ }^{21}$ Rask-Madsen, C., and G. L. King. Vascular complications of diabetes: mechanisms of injury and protective factors. Cell Metab. 17:20-33, 2013. https://doi.org/10.1016/j.cmet. 2012.11.012.

${ }^{22}$ Rocha, L. A., D. A. Learmonth, R. A. Sousa, and A. J. Salgado. alphavbeta3 and alpha5betal integrin-specific ligands: from tumor angiogenesis inhibitors to vascularization promoters in regenerative medicine? Biotechnol. Adv. 36:208227, 2018. https://doi.org/10.1016/j.biotechadv.2017.11.004.

${ }^{23}$ Shin, D., et al. Expression of ephrinB2 identifies a stable genetic difference between arterial and venous vascular smooth muscle as well as endothelial cells, and marks subsets of microvessels at sites of adult neovascularization. Dev. Biol. 230:139-150, 2001.

${ }^{24}$ Sun, X., W. Altalhi, and S. S. Nunes. Vascularization strategies of engineered tissues and their application in cardiac regeneration. Adv. Drug Deliv. Rev. 96:183-194, 2016. https://doi.org/10.1016/j.addr.2015.06.001. 
${ }^{25}$ Sun, X., S. Evren, and S. S. Nunes. Blood vessel maturation in health and disease and its implications for vascularization of engineered tissues. Crit. Rev. Biomed. Eng. 43:433-454, 2015. https://doi.org/10.1615/CritRevBiomed Eng.2016016063.

${ }^{26}$ Sun, X., and S. S. Nunes. Overview of hydrogel-based strategies for application in cardiac tissue regeneration. Biomed. Mater. 10:034005, 2015. https://doi.org/10.1088/ $1748-6041 / 10 / 3 / 034005$.

${ }^{27}$ Taniyama, Y., et al. Therapeutic angiogenesis induced by human hepatocyte growth factor gene in rat diabetic hind limb ischemia model: molecular mechanisms of delayed angiogenesis in diabetes. Circulation 104:2344-2350, 2001.
${ }^{28}$ Wythe, J. D., et al. ETS factors regulate Vegf-dependent arterial specification. Dev. Cell 26:45-58, 2013. https://doi. $\operatorname{org} / 10.1016 /$ j.devcel.2013.06.007.

${ }^{29}$ Zhang, Y. S., et al. 3D bioprinting for tissue and organ fabrication. Ann. Biomed. Eng. 45:148-163, 2017. https://d oi.org/10.1007/s10439-016-1612-8.

Publisher's Note Springer Nature remains neutral with regard to jurisdictional claims in published maps and institutional affiliations. 